\title{
Quantitative relations between visual search speed and target-distractor similarity
}

\author{
DONALD S. BLOUGH \\ Brown University, Providence, Rhode Island
}

\begin{abstract}
Three pigeons worked on visual search tasks in which arrays of 32 small forms appeared in computer-driven displays. Their task was to peck at a unique target among identical distractors; the main dependent variable was the speed with which this target was found. The target and distractor forms for each trial were chosen from a set of forms (usually 16); in most experiments, all possible pairs of forms appeared in each experimental session. Six experiments were run; each yielded a matrix of mean reaction times (RTs), one for each of the form pairs. Such matrices were subject to multidimensional scaling, from which best-fitting interform distances were determined. In four experiments with disparate form sets, Euclidean distance between pairs of forms in multidimensional space was found to approximate an exponential decay function of $\mathrm{RT}-K$, where $K$ was a constant set for each subject. Two additional experiments showed that RT distributions for single-form pairs were well fit by the convolution of a fixed distribution, approximately Gaussian, and an exponential distribution whose decay parameter varied with interform similarity. The exponential decay parameter may be taken to reflect momentary detection probability, and interform distance in multidimensional space may be taken to measure similarity. Thus, the data as a whole suggest an exponential relation between the probability of detecting a target and the similarity of that target to its surrounding distractors. This relationship is analogous to Shepard's law of generalization, which states an exponential relation between response probability and similarity.
\end{abstract}

Stimulus similarity is important to most psychological research, if only because we wish to specify the range of stimulus conditions over which any finding may hold. The similarity of visual forms is currently of particular interest in the context of research on pattern recognition. However, there are rather few data relating quantitative measures of similarity to measures of form perception. This article attempts to provide such data for the relation between similarity and the speed of visual search, using pigeons as subjects. Two sets of experiments are described. The first relates target-distractor similarity to mean search reaction time (RT) for several form sets. The second attempts to identify the component of RT that is affected by target-distractor similarity.

The first set of experiments follows Shepard (e.g., 1957) and others in representing similarity between two stimulus objects as a distance in psychological space between points representing those objects. This approach bypasses the psychophysics of similarity, in that no relationship between physical stimulus properties and distances or response measures need be assumed or determined. Arguing from theoretical and empirical considerations, Shepard (e.g., 1958) proposed that when the response measure is conditional probability (relative frequency), then a single similarity function, or generalization gradient, holds across all stimulus sets. This is

This research was supported in part by National Science Foundation Grant BNS-8025515. Address correspondence to Donald S. Blough, Department of Psychology, Brown University, Providence, RI 02912. an exponential decay function, or something close to it. The supporting data sets include several from pigeon generalization experiments (Shepard, 1965). Recently, Shepard $(1986,1987)$ derived the exponential generalization function from a new set of fundamental assumptions about the decision process.

Less well documented are generalization functions relating similarity to measures other than relative response probability. Some data suggest the exponential function for similarity ratings (e.g., Ekman, 1954). Shepard (1981) indicated that discriminative RT may bear a reciprocal relation to similarity. To my knowledge, no function relating search speed to similarity has been proposed, though visual search is known to be affected by the similarity among forms in the searched display in humans (e.g., Estes, 1972) and pigeons (e.g., D. S. Blough, 1979, 1984, in press; D. S. Blough \& Franklin, 1985; P. M. Blough, 1984). Nor have the various models of search (e.g., Harris, Shaw, \& Altom, 1985; Harris, Shaw, \& Bates, 1979; Hoffman, 1979) attempted to formalize the similarity-speed relationship.

The multidimensional scaling procedure used here to scale similarity assumes that the time taken to find a particular visual stimulus in a multiple-item display increases monotonically with the similarity between that stimulus and surrounding forms. The term "similarity" seems appropriate, rather than "discriminability," for in this task various pairs of quite different forms often yield different search speeds. In the first set of experiments, the primary datum was the mean of many RTs with a given 
form (the "target") surrounded by many copies of another object (the "distractor"). Such means were collected for many pairs of forms. In each experiment, a set of forms was presented in all possible target-distractor combinations. The resultant set of mean RTs yielded, through multidimensional scaling, a function relating similarity to search RT. For all of the experiments, this function proved to approximate an exponential decay function, once a constant had been deducted from RT.

To explore the nature of the RT measure in greater depth, a second set of experiments generated detailed RT distributions for a few target-distractor pairs of differing similarity. The resultant RT distributions could be fit, to a good approximation, by the convolution of a constant distribution (either Gaussian or empirically determined) with exponential density functions varying in their decay constants. This fit suggests that the exponential function relating $\mathrm{RT}$ to similarity may be stated in a more fundamental way: The momentary probability of finding an object is an exponential function of the similarity of that object to its background. The data as a whole thus suggest that a variant of Shepard's law of generalization may be extended to visual search.

These experiments use the odd-item search method (D. S. Blough, 1986): In a display with one unique target and many identical distractors, the subject's task is to find the target. All possible pairings (as targets and distractors) of a rather large set of forms appear in each session. With well over 1,000 trials run per session, this method generates the large number of RTs that are required for quantitative analysis. Though odd-item search is conceptually a simple procedure, its implementation as an operant conditioning task involves a number of technical aspects. The method has been set forth in detail elsewhere (D. S. Blough, 1986) and only major aspects are described here.

\section{GENERAL METHOD}

\section{Subjects}

The same 3 male White Carneaux pigeons (Columba livia) served as subjects in all experiments. The birds had been used previously in a number of search tasks (e.g., D. S. Blough \& Franklin, 1985). The duration of food presentation during experimental sessions was adjusted for each bird to maintain its weight at approximately $80 \%$ of its free-feeding weight; durations ranged from 1.8 to $2.5 \mathrm{sec}$ per reinforcement.

\section{Apparatus and Stimuli}

The apparatus and display characteristics have been described in detail elsewhere (D. S. Blough, 1986). Briefly, birds were run in a standard operant chamber. Directly above a centrally located feeder was the face of a black-and-white display monitor, on which search displays appeared in a visible area of $10.5 \times 6 \mathrm{~cm}$. Six response locations were defined across the display, each consisting of a vertical stripe $1.4 \mathrm{~cm}$ wide in which was centered one column of four display forms. The occurrence of pecks to the screen in each of these areas was sensed by an infrared detector system (Figures 1 and 2 of D. S. Blough, 1986). These arrangements are depicted in Figure 1. The chamber was illuminated by a dim over-

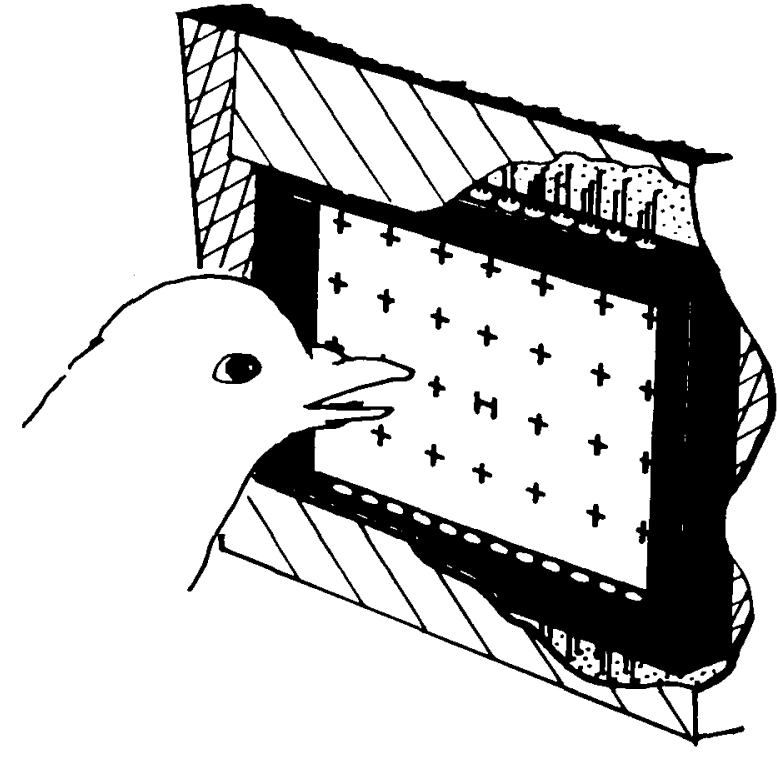

Figure 1. Display screen and response sensing array, showing a typical odd-item display. (From "Odd-Item Search by Pigeons: Method, Instrumentation, and Uses" by D. S. Blough, 1986, Behavior Research Methods, Instruments, \& Computers, 18, p. 414. Copyright 1986 by the Psychonomic Society, Inc. Reprinted by permission.)

head light and white masking noise was provided. An Atari 800 personal computer produced the display, controlled the experiment, and recorded response $\mathrm{RTs}$.

The display on a given trial consisted of an array of 32 black forms in four rows and eight columns. One of these forms, the "target," was unique; it could appear in either of the middle two rows and any of the middle six columns of the display. Thirty-one identical copies of another form, the "distractor," filled all remaining locations in the display (see examples in Figures 1 and 3). The pair of forms used on each trial was drawn from a larger set, which differed for each experiment.

\section{Procedure}

Each trial began with the appearance of a stimulus matrix on the previously blank white monitor screen. If two successive pecks to the target location occurred within $8.5 \mathrm{sec}$ after the display appeared, the forms briefly changed color from black to white and disappeared, while the feeder light came on for $1 \mathrm{sec}$. On $7 \%$ of such trials, the feeder light stayed on and food was presented. A 2-sec intertrial interval followed. An RT was recorded if the trial was not a correction trial, as specified below. A new combination of forms appeared on the next trial.

If the pigeon failed to peck the target twice in succession within $8.5 \mathrm{sec}$, the trial terminated after two pecks to any location, the screen went blank, and a "miss" was recorded. One or more correction trials followed a miss. The first correction trial was a representation of the missed display. After a second miss, the target appeared on the screen with a set of extremely different distractors, either four black dots in each form position or a large gray square in each form position. Such trials invariably elicited a correct response within $8.5 \mathrm{sec}$, and testing then proceeded with a new display.

On each trial of a given experiment, 2 forms were chosen from the set used in that experiment. Each form in the set appeared as a target equally often in every block of 32 trials (for sets of 16 forms) or 12 trials (for sets of 4 or 6). Every possible combination of 2 
different forms appeared equally often during each session, and the target for each combination was equally often placed in each of the six target location areas.

\section{Data Analysis}

Data were combined across a number of sessions as specified for each experiment. The performance of the birds was indicated by the following statistics, determined for each bird's data and for the mean across birds: Percent correct responses across all stimulus pairs, proportion of stimulus pairs yielding more than $75 \%$ correct responses, mean RT for each stimulus pair, and split-half (alternate block) reliability of $\mathrm{RT}$. Interbird correlations were also determined.

Reduction of the RT data was complicated by the fact that for quite similar forms, search was sometimes not completed within $8.5 \mathrm{sec}$ after trial onset, and a miss was recorded. Omission of such misses from the calculation of mean RT led to an underestimation of the value that would have been obtained had each trial continued indefinitely. Thanks to the regularity of the RT distributions (Experiment 2), it was possible to estimate the mean of these missing RTs for each stimulus pair (see Appendix). However, points for those few stimulus pairs that were missed on more than $25 \%$ of trials were excluded from the RT-similarity functions presented below.

Matrices of mean RTs were input to ALSCAL, a multidimensional scaling program run under the SAS system (SAS Institute, 1982). Nonmetric ("ordinal") and metric ("interval") options were used as stated below, as was the INDSCAL variation, which uses individual subject differences to advantage. The fits of Euclidean distances to optimally scaled data are stated in terms of variance accounted for $\left(r^{2}\right)$ and Kruskal stress, Formula 1 (stress). For most of these purposes, each bird's RT data were transformed as follows:

$$
T=\log (\mathrm{RT}-K),
$$

where $T$ is the transformed value, $\mathrm{RT}$ is the bird's mean reaction time for a given target-distractor combination, and $K$ is a constant equal to the bird's minimum RT in a given experiment, less $0.05 \mathrm{sec}$. The constant $K$ represents the commonly accepted "irreducible minimum" RT that is assumed invariant with stimulus factors, but which varies across subjects; the specific value chosen for $K$ depended on results from Experiment 2 (see Appendix). Equation 1 thus provides a way to make the data from different subjects comparable before combining them. Also, this transformation of RT turned out to be approximately linear with similarity, a matter discussed at length below. Prior to scaling, each data matrix was also "folded" by averaging the matrix with its transpose. Each resulting value was the mean of two values, one for each member of a stimulus pair appearing as target with the other as distractor.

\section{EXPERIMENT 1A}

This experiment considered search among simple forms differing only in size. It provided a relatively uncomplicated look at similarity in a putatively one-dimensional case, and also served as a prototype for the subsequent studies. The stimuli were six black squares of different sizes. Each of the six squares was paired with every other square; the bird had to find the different-sized square in each display.

\section{Method}

The experiment followed the general method outlined above, with additional details as follows. The six stimulus forms appear in Figure 2. The sides of the squares varied from $1.2 \mathrm{~mm}$ ( 3 pixels)

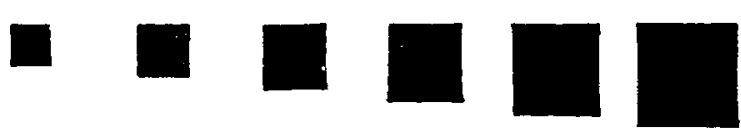

Figure 2. The set of forms used in Experiment 1A. Relative size is to scale; the largest form was actually $3.2 \mathrm{~mm}$ square.

to $3.2 \mathrm{~mm}$ ( 8 pixels) in steps of $0.4 \mathrm{~mm}$. The squares filled the standard $8 \times 4$ display matrix described above, except that their positions were randomly perturbed within rows and columns. Each form could appear at one of four positions, such that it was centered at one corner of an imaginary square $3.2 \mathrm{~mm}$ on a side. A typical display appears in Figure 3.

Each square was paired as target 43 times with every other form as distractor in every session, for a total of 1,290 trials per session. The birds were run for nine sessions, of which the last six provided the data reported below; thus, each bird contributed 258 RTs for each target-distractor pair.

\section{Results and Discussion}

Data were analyzed as described above. Mean percent correct responses for the 3 birds were 93.5, 97.3, and 89.3. Of the 30 stimulus pairs, $28(93 \%)$ yielded correct responses within $8.5 \mathrm{sec}$ on more than $75 \%$ of trials. Splithalf reliability for the mean RT data was .996 . Mean interbird correlation was .975 .

For the simplest and most direct look at distance configurations for these data, RT data were combined across birds and folded, but the log transformation described above (Equation 1) was omitted. The data were then fit with the nonmetric (ordinal) version of ALSCAL in one and two dimensions, with the results shown in Figures 4A and 4B. As one would expect with so few data points, the fit was good in both cases (stress $=.01$ and .003 , $r^{2}=1.0$ ).

The tendency of scaling algorithms to bend an essentially one-dimensional relation into a C-shaped curve has been noted previously. Shepard (1974) argued that often in such cases the underlying one-dimensional structure is distorted by noisy data. This is probably the case here, and the use of an extra dimension appears to clarify the data in the experiments below as well. A fuller account of this matter is given in the Appendix.

Figure 5A displays a point for each pair of stimuli, with mean RT on the ordinate and distance, as shown in Figure 4B, on the abscissa. The curve through the points,

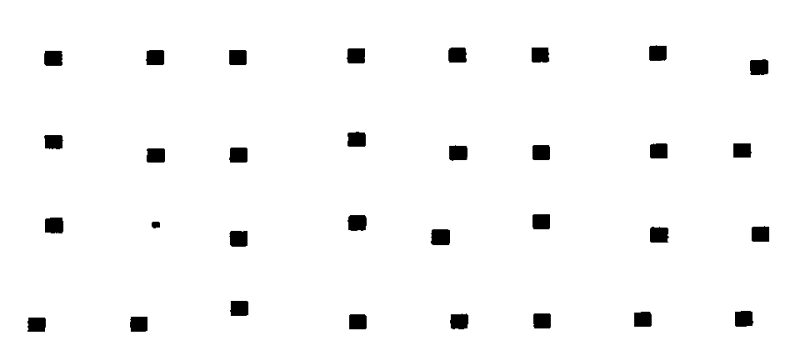

Figure 3. A typical display for Experiment 1A. Two forms appear, one singly as target, the other repeated as distractor. Targetdistractor pairs were chosen randomly from the set shown in Figure 2. 
$\underline{A}$

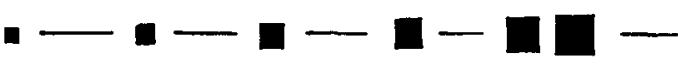

B

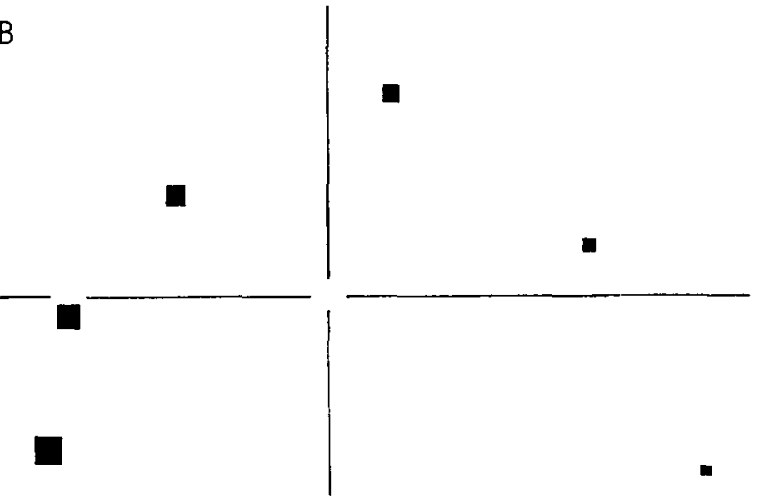

Figure 4. A: One-dimensional ALSCAL representation derived from RTs in Experiment 1A. Mean RTs were not transformed before input. B: Same, two-dimensional configuration.

drawn by eye, appears approximately exponential. This appearance is confirmed by a similar plot based on data subjected to the log transformation shown in Equation 1 (see also Appendix). The exponential function is indicated by the linear relation between the transformed data and distance. The lack of scatter of the points in Figure 5B is doubtless due in part to the fact that these data were exponentially transformed before input to ALSCAL; a good fit to relatively few points may demand little rescaling. Thus, larger data sets are necessary to be more certain of the scaling outcomes, and such sets were generated in the next three experiments.

\section{EXPERIMENT 1B}

The results of Experiment 1A suggested an exponential relationship between pigeon visual search time and target-distractor similarity. The following three experiments provided further information on this relationship, with larger form sets selected to emphasize differing aspects of stimulus dimensionality. (The psychophysics and dimensional structure of these stimuli require fuller treatment, which is reserved for a future publication.)

Experiment 1B used a set of forms that expanded both conceptually and numerically upon the forms used in Experiment $1 \mathrm{~A}$. The stimuli were 16 rectangles varying in two dimensions-height and width. To minimize perceptual discontinuity, all of the rectangles were wider than any was high. Estimates of the similarity of such rectangles have been obtained from humans in several experiments (e.g., Krantz \& Tversky, 1975; Monahan \& Lockhead, 1977; Wender, 1971; Wiener-Ehrlich, 1978). It has been argued that, for humans, rectangles exemplify "integral," holistically processed stimuli, from which distinct dimensions (e.g., height and width) are not perceptually extracted (e.g., Wiener-Ehrlich, 1978), although this conclusion has been questioned (e.g., Schönemann, Dorcey, \& Kienapple, 1985).

\section{Method}

The general method described above was followed. The set of stimulus rectangles is shown in Figure 6 . The largest rectangle mea-
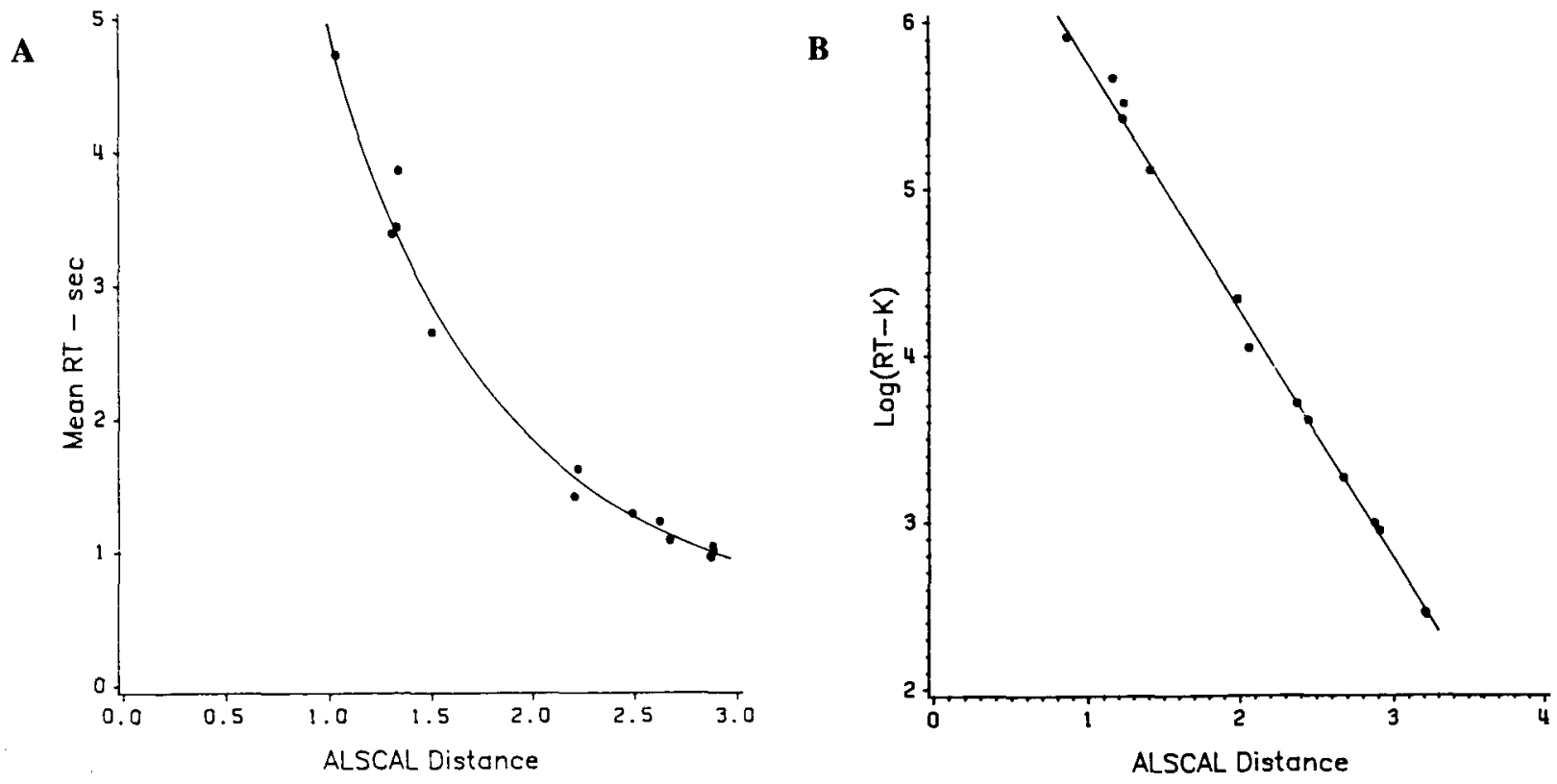

Figure 5. A: Distances between the points shown in Figure 4B (data of Experiment 1A), plotted against the corresponding RT values. B: Distances from comparable two-dimensional scaling of the same RTs transformed according to Equation 1. The numbers on the ordinate are natural logs of time in milliseconds. 


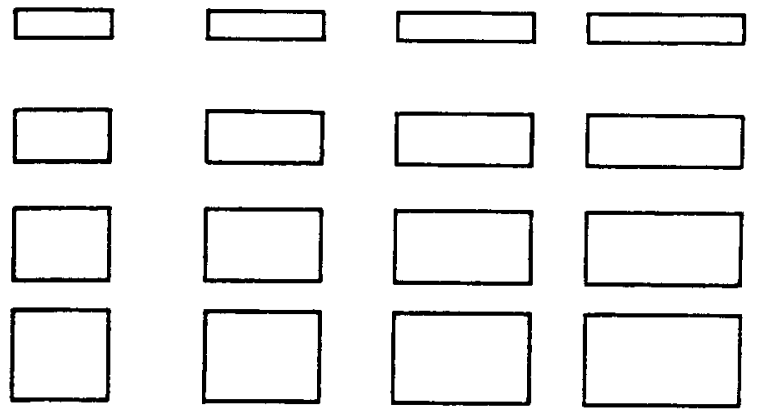

Figure 6. The set of stimulus forms used in Experiment 1B; the rectangles vary orthogonally in width and height.

sured $6.4 \times 3.6 \mathrm{~mm}$ on the display screen, and the smallest measured $4.0 \times 1.2 \mathrm{~mm}$. Figure 6 shows the relative proportions of the other rectangles. The 16 stimuli yielded 240 different target distractor pairs. In every daily session, each such pair appeared six times, for a total of 1,440 trials. Each of the 3 birds was run for 15 sessions; data from the last 12 of these are reported here. Thus, the results from each bird were based on 72 trials with each targetdistractor pair.

\section{Results and Discussion}

The data were analyzed as described under General Method. Mean percent correct responses was $89.2 ; 83 \%$ of stimulus pairs were searched correctly on $75 \%$ or more of trials. Split-half reliability of mean RT data was .993, and mean interbird correlation was .955 . The mean, transformed, folded RT data were scaled in four, three, and two dimensions with ALSCAL (ordinal option). The configuration found with two dimensions assumed a $\mathrm{C}$ shape like that in Figure 4B, and as in Experiment 1A, this probably reflects variance at the extreme distances. The distance along this $\mathrm{C}$-shaped line was dominated by the vertical dimension of the rectangles. The relative contribution of the stimulus variables is better represented by two of the three dimensions found in three-dimensional scaling. This result appears in Figure 7; here, stress $=.021$ and $r^{2}=.998$. The details of this configuration are not our major interest, but several aspects may be noted. The stimulus variations in vertical and horizontal extent interacted markedly. The vertical changes were dominant, with the horizontal playing an increasing role with the larger forms. Such interactions, although less extreme, characterize human judgments of rectangles (e.g., Wiener-Ehrlich, 1978), supporting the notion that for pigeons, as for humans, the stimuli are integral and are not parsed into dimensions in this task.

Our central interest here is the function relating interstimulus distance to RT. Despite the fact that arbitrary rescaling of the $\log (\mathrm{RT}-K)$ data was permitted, ALSCAL made only minor shifts to achieve a fit, and a regular relationship emerged from scaling in two, three, and four dimensions. The function relating distance and $\log (\mathrm{RT}-K)$ appears for three dimensions in Figure $8\left(r^{2}=.995\right.$, stress $=.025$ ). The essential linearity of the relationship implies an exponential function relating distance and RT; however, this is modified in the present case by a slight negative curvature.

\section{EXPERIMENT 1C}

The exponential relation between search RT and form similarity, suggested in Experiment 1A, was supported by scaling results from the "integral" stimulus rectangles used in Experiment 1B. Experiment 1C investigates this relationship with stimuli defined by two "separable" dimensions. Stimulus aspects like those used in the first two experiments were combined in a manner that favors their separate processing. One might expect Euclidean scaling (as with ALSCAL) to be less successful with data

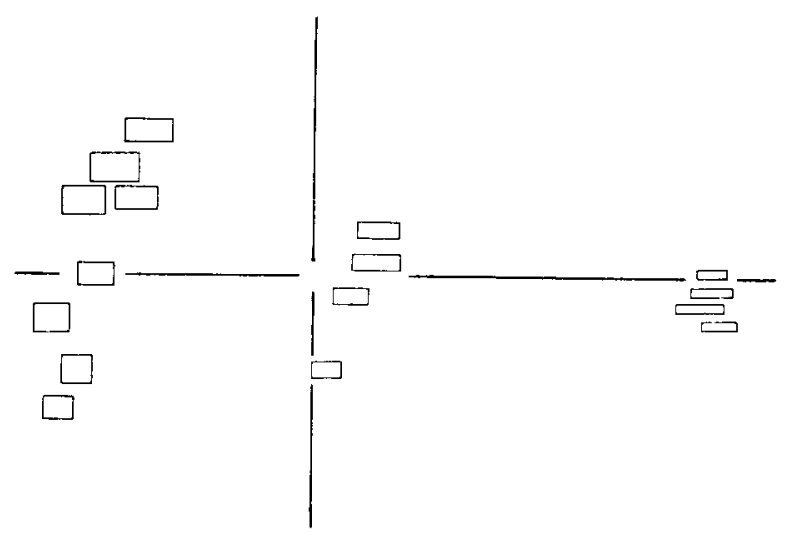

Figure 7. Two-dimensional representation derived by ALSCAL from RT results of Experiment 1B. Monotonic rescaling of the input data was permitted (ordinal scaling option).

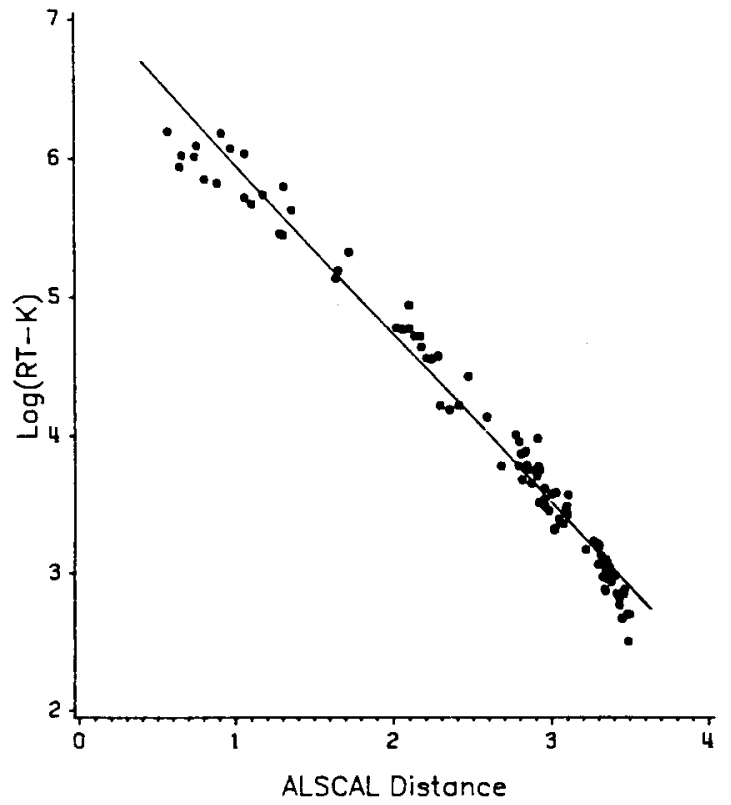

Figure 8. Distances derived from three-dimensional scaling of the data of Experiment 1B, plotted against their corresponding $\log (\mathrm{RT}-K)$ values. The straight line was fit by eye. 


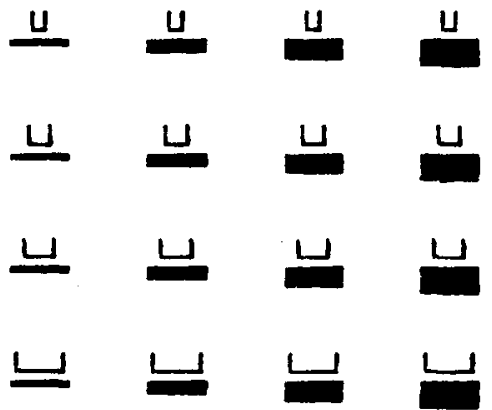

Figure 9. The forms used in Experiment 1C; two separable dimensions, the width of the upper segment and the size of the lower block, vary orthogonally.

based on such stimuli, since they are predicted to follow a "city-block" metric (e.g., Shepard, 1964). This turned out not to be the case here (see below, and Appendix).

\section{Method}

The general method described above was followed. Sixteen stimulus forms were each composed of a U-shaped line above a black rectangle; four Us of different widths were combined in all possible combinations with four different rectangle heights, as shown in Figure 9. The black rectangle was $4.5 \mathrm{~mm}$ wide, and the other dimensions were scaled to this as shown in the figure. The number and composition of daily sessions were the same as in Experiment $1 \mathrm{~B}$.

\section{Results and Discussion}

The data were analyzed as described under General Method. Mean percent correct responses was $96.9 ; 97 \%$ of stimulus pairs yielded correct responses on $75 \%$ or more of trials. Split-half reliability of mean RT data was 979. Mean interbird correlation was .765 , notably lower than in the previous experiments. This reduced intercorrelation is consistent with the analyzable or separable nature of the stimuli used in this experiment, thus suggesting that different subjects selectively attended more to one or the other of the stimulus aspects (Shepard, 1964). In any case, the individual differences prompted scaling both with ALSCAL, as above, and with INDSCAL, which uses individual differences to find nonarbitrary dimensional axes. INDSCAL also provides different weights for each subject on each dimension. As it happened, the configurations yielded by the two methods did not differ substantially, so the ALSCAL results, comparable to those above, are reported here.

A two-dimensional configuration from ALSCAL (ordinal fit) appears in Figure $10\left(r^{2}=.957\right.$, stress $\left.=.088\right)$. It is evident that this representation organizes the stimuli along dimensions consistent with their two physical components. Because of the apparent separability of these components, it is worth mentioning that city-block fits were comparable to Euclidean fits in two dimensions and somewhat less good in three and four dimensions (see Appendix for details). As in the previous experiments, at least one more dimension is needed to accommodate the curl of the extremely dissimilar stimuli back toward each other.
All dimensionalities revealed a generally exponential form of the similarity versus RT function, as shown with relatively little noise for the four-dimensional case in Figure $11\left(r^{2}=.991\right.$, stress $\left.=.030\right)$.

\section{EXPERIMENT 1D}

The three previous experiments used stimulus sets putatively composed of, respectively, one dimension, two integral dimensions, and two separable dimensions. This experiment extends the nature of tested stimuli to a set defined by the presence or absence of four binary features.

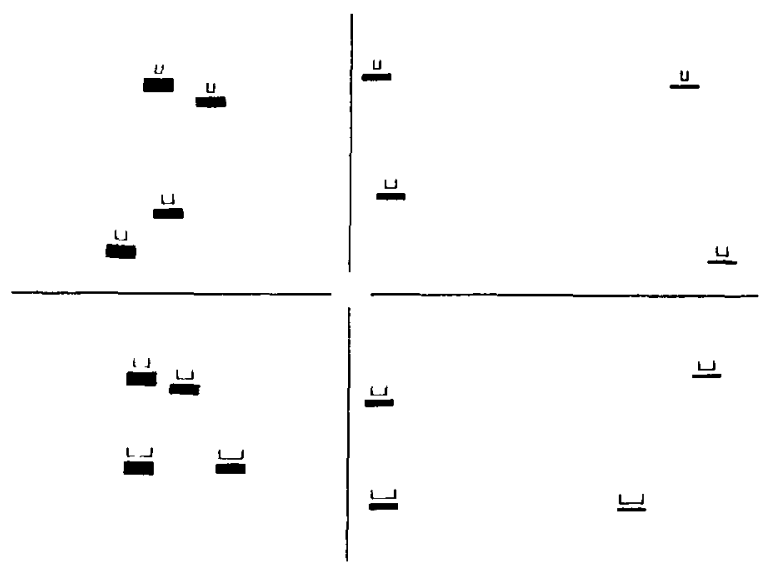

Figure 10. Two-dimensional representation derived by ALSCAL from RT results of Experiment 1C.

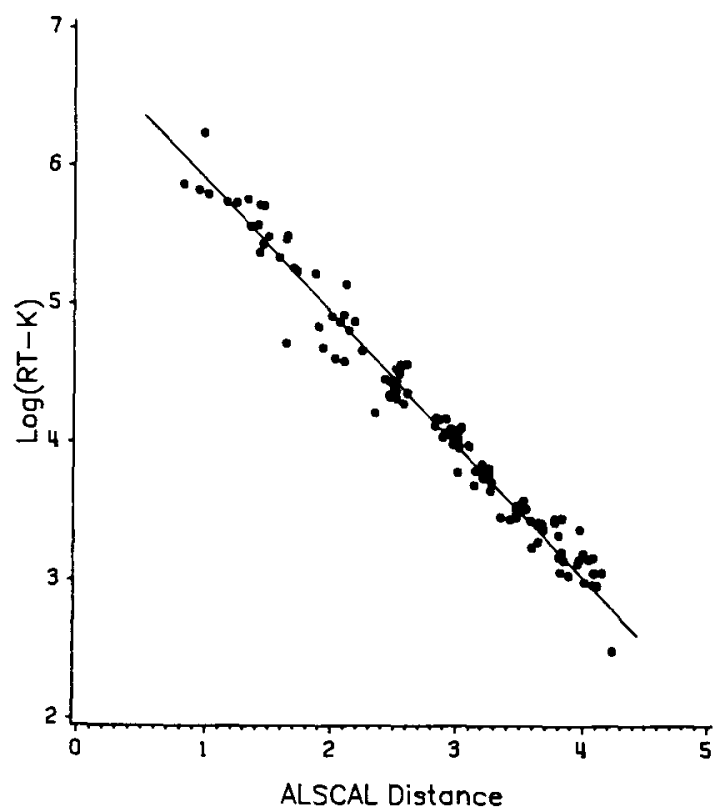

Figure 11. Distances derived from four-dimensional scaling of the data of Experiment $1 \mathrm{C}$, plotted against the corresponding $\log (\mathrm{RT}-K)$ values. The straight line was fit by eye. 


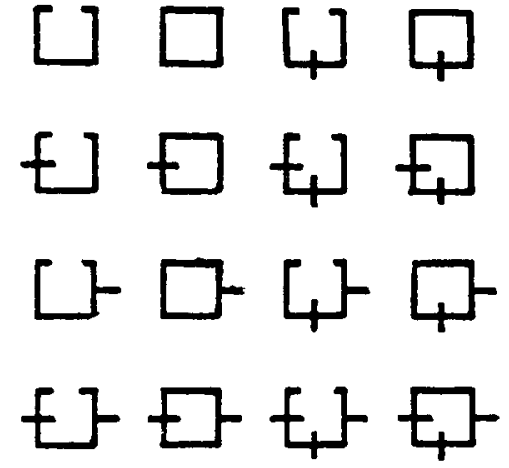

Figure 12. The forms used in Experiment 1D. The forms consist of a U-shaped line to which are added from zero to four line segments in different locations.

\section{Method}

The general method described above was followed. The set of stimulus forms is shown in Figure 12. All the forms included a $U$ shape; they differed by the presence or absence of four short line segments, three horizontal and one vertical, which appeared in all possible combinations. The $U$ form was $3 \mathrm{~mm}$ on a side.

For reasons irrelevant to the present analysis, the birds had two series of runs with these forms, one of 17 sessions and the second of 12 sessions, separated by other training. The data reported here are from the second series of 12 sessions. Session length and trial organization were as described under General Method and under Experiment 1B.

\section{Results and Discussion}

The data were analyzed as described under General Method. Mean percent correct responses was $96.2 ; 93 \%$ of stimulus pairs yielded successful searches on $75 \%$ or more of trials. Split-half reliability of mean RT data was .990 , and mean interbird correlation was .889. As in Experiment $1 \mathrm{C}$, the somewhat low interbird correlation prompted the use of both INDSCAL and ALSCAL. Again there was little substantive difference in the two configurations, but INDSCAL provided a meaningful orientation of the dimensional axes. A two-dimensional INDSCAL outcome appears in Figure $13\left(r^{2}=.857\right.$; stress $=.154)$. It is evident that presence versus absence of the left line segment defines the horizontal dimension. The lower line segment similarly defines the vertical dimension and the other segments control local clustering. In three dimensions, INDSCAL found the top horizontal segment to control the third dimension. Interestingly, searches for any target that differed from a distractor only in the presence or absence of the right line segment were extremely difficult, accounting for almost all errors and very long RTs.

Again, in all dimensionalities, the similarity versus RT function followed an exponential trend. An ALSCAL result based on four dimensions appears in Figure 14 $\left(r^{2}=.990\right.$, stress $\left.=.030\right)$.

\section{EXPERIMENT 2A}

Experiments $1 \mathrm{~A}$ through $1 \mathrm{D}$ indicate that for several sorts of stimulus forms, the time taken in odd-item search is approximately an exponential function of the similarity between the target and distractor forms, after a constant has been subtracted from all RTs. The following experiments sought a deeper interpretation of this function through exploration of RT distributions, which led to the suggestion that the reciprocal of mean search RT (beyond a constant component) approximately estimates the instantaneous probability that a target will be found among copies of a distractor.

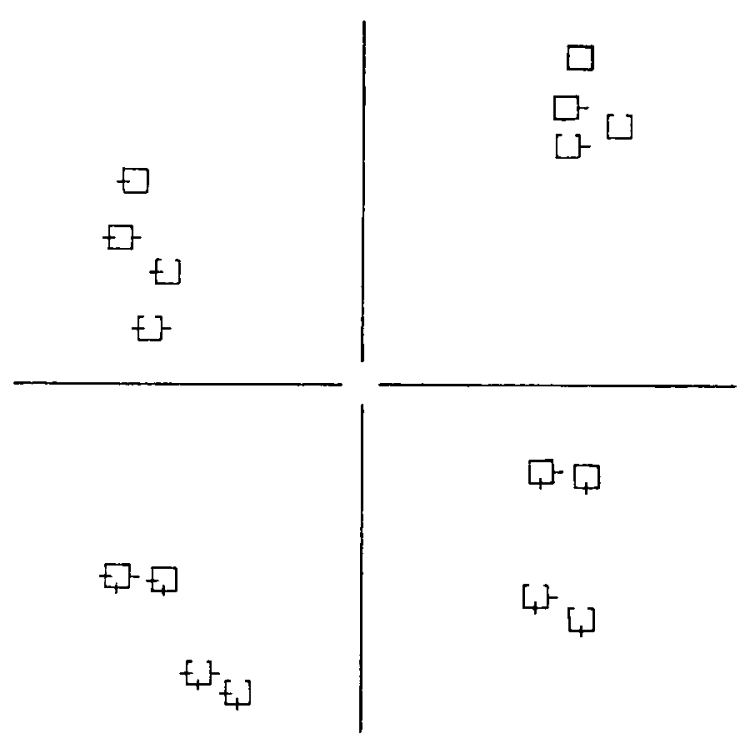

Figure 13. Two-dimensional representation derived by INDSCAL from RT results of Experiment 1D. The presence or absence of a line segment at the left or at the bottom of the form define the dimensional axes.

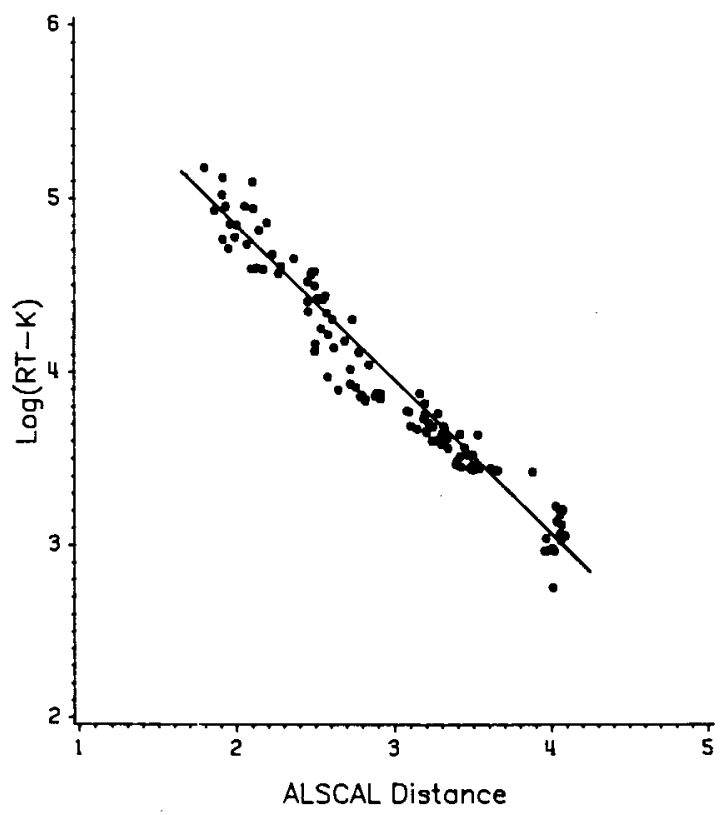

Figure 14. Distances derived from four-dimensional scaling of the data of Experiment 1D, plotted against the corresponding $\log (\mathrm{RT}-K)$ values. The straight line was fit by eye. 
The experiments were similar to Experiment 1A, except that a very large number of trials was run with each of a few target-distractor combinations in order to develop detailed RT distributions.

\section{Method}

The procedure followed that of the general method described earlier and Experiment $1 \mathrm{~A}$, except in the following particulars. The four smallest forms from Experiment $1 \mathrm{~A}$ served as stimuli. Instead of using all possible combinations of these four forms, only those combinations were used in which a larger form appeared as a target on the background of a smaller form; thus, there were only six trial types. This set was designed to yield a few target-distractor combinations across a range of similarities, to the exclusion of extreme similarities that would generate errors. As before, the target could appear in any of 12 locations, and the remainder of the display was filled with 31 copies of the distractor. The birds were run for 12 sessions, of which the last 11 supplied data for analysis. Each session included 1,188 trials, with each target-distractor combination appearing 198 times. The final data for 11 sessions included $2,178 \mathrm{RTs}$ for each target-distractor combination.

Data analysis differed from that in Experiments 1A-1D, for here the basic data were RT distributions for a few stimulus pairs, instead of mean RTs for many pairs. RTs for each bird and pair, across all sessions, were distributed into $1 / 30^{-}$-sec bins, with the few errors counting as 8.5-sec RTs.

For some analyses, RT distributions were combined across subjects in the manner recommended by Ratcliff (1979) for RT data. The combination process was equivalent to "Vincentizing": The 2,178 RTs for each stimulus pair and bird were ordered from least to greatest, and the ordinally corresponding RTs were averaged across birds. The resulting mean RTs were distributed into $1 / 30-\mathrm{sec}$ bins just as though they had come from one subject. The analyses next to be described were performed on the individual and the group results; since the group analyses appeared to well represent all key features of the individual data and were a bit smoother, they are shown here for simplicity.

The RT distributions were fit by combinations of underlying distributions that varied in few parameters. Fits were done by a hillclimbing procedure, seeking maximization of $\omega^{2}$ through gradual shifts in the parameters.

\section{Results and Discussion}

As planned, error rates were very low, with an overall mean percent correct of 99.7 . Thus, a negligible proportion of RTs were clumped at the 8.5-sec cutoff.

The RT distributions were unimodal with a skew qualitatively resembling RT data from many tasks with humans. The combined distributions for the six stimulus conditions appear in Figure 15 (black dots).

The appropriate mathematical description of RT distributions has occasioned much debate. A convenient tabulation of major alternatives is provided by Burbeck and Luce (1982), and extensive discussion is given by Luce (1986). Familiar unitary distributions (such as Poisson) neither fit the present data nor recommend themselves on rational grounds, and most investigators assume that RTs reflect a minimum of two groups of processes operating serially (McGill, 1963). Lumped together in one group are processes such as neural transmission and motor response, which together contribute a duration that is essentially independent of stimulus conditions. The other group includes perceptual and decision processes, which contribute durations that vary with stimulus parameters. Hohle (1965) suggested that the former component might have a Gaussian distribution, and the latter an exponential distribution. If this were true, an observed RT distri-
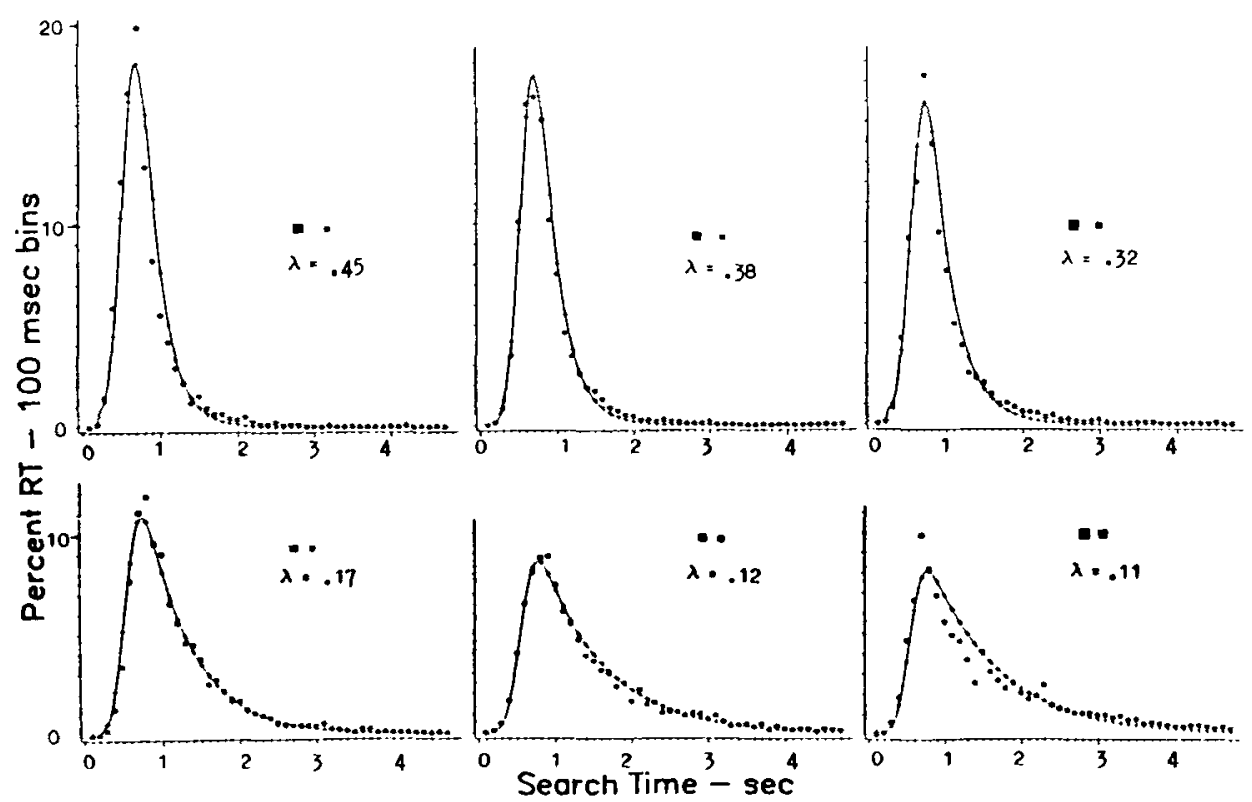

Figure 15. Separate points represent RT frequency distributions for the six target-distractor combinations used in Experiment $2 \mathrm{~A}$. The fitted curves represent the convolution of two distributions, a Gaussian (the same for all fits) and an exponential with the decay constant indicated. Omega-squared, the measure of goodness of fit (variance accounted for) exceeded $\mathbf{9 9 9 9}$ for all fits. 
bution should be well fit by the convolution of Gaussian and exponential functions, specified by three parameters: the mean and the variance of the Gaussian, and the decay constant of the exponential. This convolution has subsequently been strongly advocated by Ratcliff and Murdock (1976) as at least a useful summary of RT data, relatively easy to fit, with rationally interpretable parameters. It was effectively used by Ratcliff in his memory retrieval model (1978) and recently by Hockley (1984) to model both memory and visual search data. Unfortunately, Hockley's task (list scanning) and independent variables are not sufficiently comparable to those in the present experiments to permit meaningful comparison of results beyond the shape of RT functions.

The Gaussian-exponential convolution seems well suited for the present case. The simplest account of search for a randomly placed target is that the target has a fixed chance of being found at any moment; this yields the exponential decay component of RT. As for the other component, the familiar assumption of many factors independently varying in time suggests the Gaussian, though there are other plausible alternatives, and the Gaussian yields some difficulties that we shall encounter below.

Fits of the data by Gaussian-exponential convolutions were derived by the hill-climbing method described above. Because we assume that the Gaussian contribution to the fit is the same for all stimulus pairs, a single Gaussian mean and variance were estimated, while a separate exponential decay constant was found for each RT distribution. The fitted functions are shown as continuous curves in Figure 15, with the numerical values of the decay parameter noted. Since only a crude iteration across the several data sets was used to fit the Gaussian parameters, it is likely that these are not quite optimal. Nonetheless, the fitted exponential decay constant was closely related to observed mean RT across the conditions; a linear relation would be expected between the reciprocal of the decay constant and mean RT if variation in the exponential component were the sole source of observed changes in mean RT. In fact, the correlation of these values was .999 .

Although the normal-exponential convolution provided a good fit to the search-time data in Experiment 2A, a systematic discrepancy appears in most instances, for the RT curve is a bit too steep to the right of its mode, and the obtained data tend to lie under the theoretical curve. (Interestingly, this is also a feature of sample RT distributions presented by Ratcliff \& Murdock, 1976.) This discrepancy appears more prominently if the RT distribution is converted to a conditional frequency distribution, or hazard function, which estimates the probability of a response at a particular time, given that no response has occurred until that time. For the Gaussian-exponential convolution, this conditional probability increases monotonically, rising at first where the Gaussian dominates, and gradually leveling off.

In contrast to the Gaussian-exponential model, the present data yielded peaked conditional functions, with the peak becoming quite prominent for easy searches (see data points in Figure 17). Such a pattern has suggested to others that the underlying decision (here, search) process might have two components. For example, an analogous pattern of RT results was found by Burbeck and Luce (1982) for human reaction times to pure tones in noise: Conditional detection probabilities for relatively strong tones peaked early and then declined, while those for relatively weak tones remained level after an initial rise. This pattern, together with other evidence, led Burbeck and Luce to support a two-process view of detection, with a "change" detector and a "level" detector operating in parallel. The possibility that something similar might occur in visual search has been suggested a number of times (e.g., Hoffman, 1979; Neisser, 1967). A familiar idea along these lines is that some sorts of targets capture attention by "popping out" from the background, while other targets, sharing (for example) crucial features with distractors, require extended serial search (e.g., Treisman \& Gelade, 1980; Yantis \& Jonides, 1984). These considerations suggest the possibility that, in the present experiments, strongly dissimilar targetdistractor pairs might invoke somewhat different processing than more similar pairs. This would complicate the interpretation of the results, and would make puzzling the apparent simplicity of the similarity-RT relation found in the first set of experiments.

However, the pattern of conditional probabilities just described does not necessarily imply the operation of two search-related processes. Although the Gaussianexponential convolution cannot account for variable peaks in the conditional probability functions, other distributions, convolved with the exponential, might do so. The Gaussian component presents other problems as well, and the next experiment was designed to produce an empirical substitute.

\section{EXPERIMENT 2B}

The data of Experiment $2 \mathrm{~A}$ were fit on the assumption that the RT component unrelated to stimulus variables has a Gaussian distribution. Although it is convenient, yields quite a good fit, and is justified in the literature, this assumed distribution for the constant RT component is somewhat unrealistic. Any process triggered by an event at time $T$ cannot produce RTs distributed indefinitely backward as well as forward from a later time $T+K$, since this implies negative RTs. Here, these impossible RTs would correspond to the left-hand tail of the Gaussian density function. Also, as we have seen, the Gaussianexponential convolution predicts a monotonically rising conditional probability function, rather than the peaked function obtained.

Experiment 2B was run under conditions designed to reduce the "search" (exponential) component of the RT to a minimal and possibly negligible value. If we continue to assume that the empirical RT distribution reflects the convolution of the exponential with a second distribution, 
the results of the experiment should provide an empirical estimate of that second distribution. This strategy has some precedent in previous RT analyses of discriminative processing. For example, in the paper already cited, Burbeck and Luce (1982) estimated the constant RT component in their sound detection task from RTs to a very large change in sound level. The present analogue to such an intense signal was a very easy search task. In Experiment 2B, black targets appeared alone on a blank white field. Presumably, relatively little time should be spent in finding the target under these conditions, and the search component of RT should be minimal.

\section{Method}

The method was similar to that employed in Experiment 2A. The three largest forms used in that experiment served as stimuli. Each of these forms appeared alone on an otherwise blank white screen, in one of the 12 central matrix positions reserved for targets in the other experiments. Each form appeared 528 times per session, and five sessions were run.

\section{Results and Discussion}

Within birds, RT distributions for the largest forms differed little, whereas RTs were slightly longer for the smallest form. Therefore, since the data were intended to estimate minimum RTs, those from the smallest form were omitted from further consideration. RTs for the two largest forms were combined within birds, and then across birds by the Vincentizing procedure described above. The resulting distribution then replaced the Gaussian in the curve-fitting process described above. That is, the newly obtained "minimum" RT distribution was convolved with an exponential distribution, and the exponential decay constant was adjusted to give the best fit to each of the six
RT distributions from Experiment $2 \mathrm{~A}$. The resulting fitted distributions are shown in Figure 16, together with the exponential decay constant. The fits are good, except for the one for the most similar target-distractor pair, which was also poorly fit by the Gaussian-exponential convolution. As before, the reciprocal of the exponential decay constants closely mirrored the mean RT of the data $(r=.999)$.

Although good, the fits shown in Figure 16 account for little, if any, more variance than the Gaussian-exponential fits described above. However, as we saw above, the Gaussian-exponential distribution had the disadvantage of a monotonically increasing conditional probability function. In contrast, the new convolutions produced conditional probability curves that roughly mirrored those from the data, moving from a markedly peaked function for dissimilar stimuli to a monotonically rising function as the exponential parameter declined. A sample of these results appears in Figure 17, with curves representing the most different stimulus pair (top) and the second most similar pair (bottom). This outcome demonstrates that the changing shape of the conditional probability function across data sets does not imply a complex search process. Thus, the data remain consistent with the idea that differences in mean RT for different form pairs flow from changes in a single variable, which can be identified with the momentary probability of target detection.

\section{GENERAL DISCUSSION}

The results of Experiments 1 and 2 jointly suggest the following formulation of the relation between similarity and the speed with which a pigeon searches an odd-item

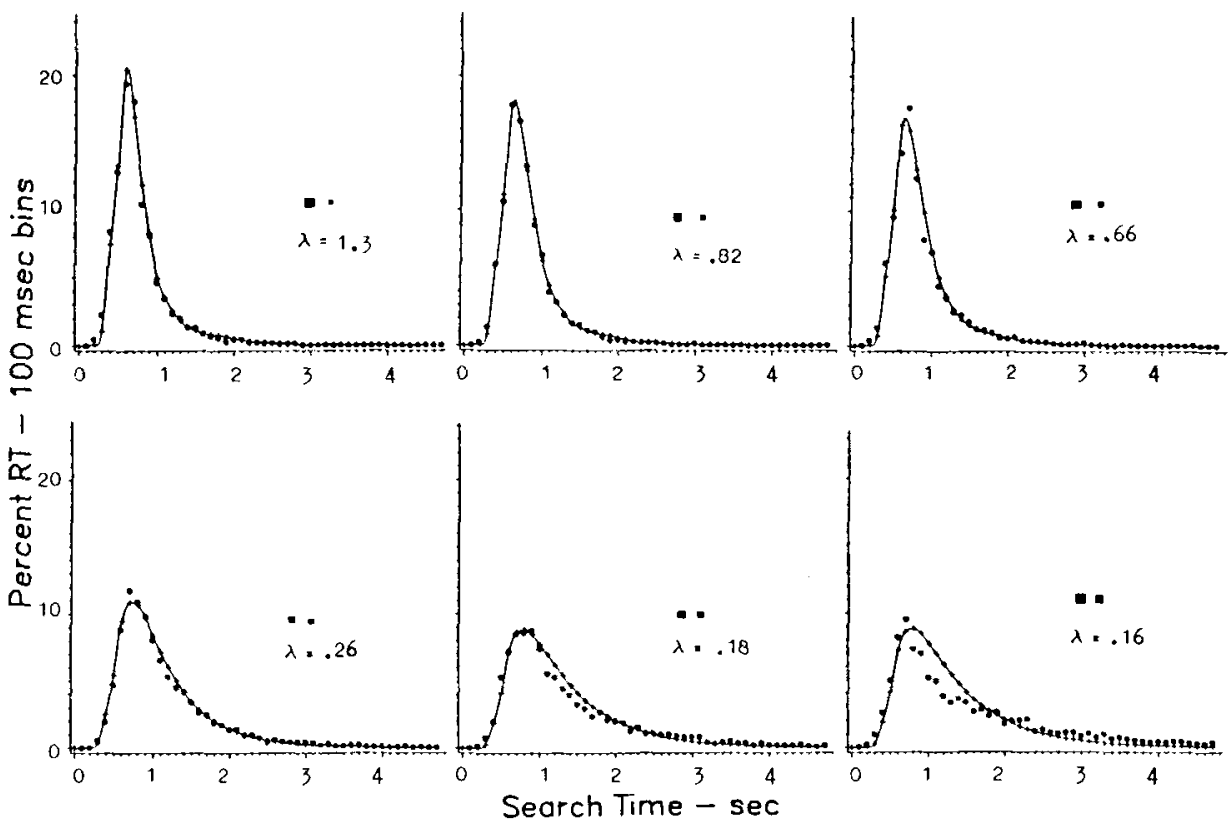

Figure 16. Empirical and fitted results of Experiment 2A. The results are the same as in Figure 15, except that a fixed RT distribution from Experiment 2B replaced the Gaussian in the derivation of fitted curves. Omega-squared again exceeded .9999 in all cases. 


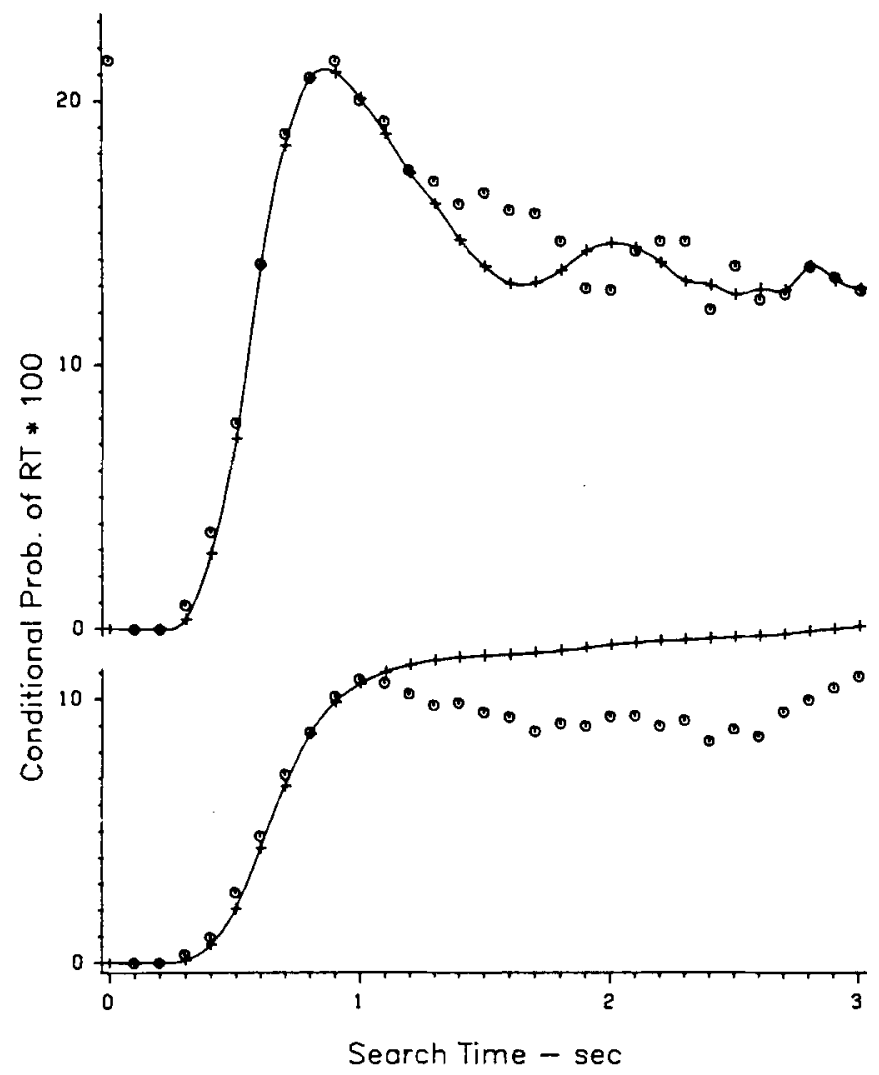

Figure 17. Curves showing the conditional probability of $R T$ as a function of search time ("hazard functions"). These functions are based on two of the panels in Figure 16. The connected points represent the theoretical fits; they show that a change in the exponential decay constant alone may change the shape of the hazard function from monotonic to nonmonotonic (see text).

display: The probability that, at any moment, a target $T$ will be found on a background of distractors $D$ is approximately an exponential decay function of the dissimilarity between $T$ and $D$, where dissimilarity is given by the Euclidean distance between $T$ and $D$ as determined by nonmetric multidimensional scaling.

If accepted, this relationship provides a tool that is potentially useful in the study of interstimulus similarity, including such classic problems as metrics and combination rules. Conversely, the relationship provides an avenue through which quantitative estimates of similarity may be introduced into models of the search process.

Before turning to such matters, we consider two possible limitations on the generality of the results. First, any measure of similarity doubtless depends to some degree on the method employed. For example, in experiments with human subjects, reaction time measures and similarity ratings have given rather different estimates of relative interform similarity (e.g., Podgorny \& Garner, 1979). Even within the search task, the manner in which stimuli are displayed could affect the results. For example, detecting a target in an array of closely spaced stimuli could result from detecting an irregularity in spacing between the target and nearby distractors, rather than form dis- similarities. Despite these cautions, there is reason to believe that the search procedure used here produces rather general results in pigeons. We have found little effect of array configuration on similarity estimates from search tasks. Also, similarities among letters of the alphabet based on search, with arrays more closely spaced than those used here, correlated highly $(.89)$ with similarities found with a discrimination-learning method (D. S. Blough \& Franklin, 1985).

A second potential problem is an apparent defect in the proposed relationship between RT and similarity. An exponential relationship predicts that as dissimilarity approaches zero, search RT approaches a rather large but certainly finite value. This implies that, given enough time, a subject should be able to find a target that is identical to the distractors in a display! This paradox may be resolved in at least two ways. First, and perhaps most likely, the exponential relationship may not hold for very small interstimulus differences. Shepard (1986) has argued that a Gaussian rather than an exponential result might be expected when a task involves extended practice and the stimuli are very difficult to discriminate. Unfortunately, the form of the RT-similarity function is difficult to determine for highly similar stimuli because, 
as errors rise, the meaning of the remaining correct RTs is difficult to interpret (see Appendix).

Possibly, the exponential RT-similarity relationship continues to hold as the target-distractor difference approaches zero. As we just saw, this implies the successful completion of searches despite target-distractor identity. However, such completion is not impossible if one allows for successful "guesses"; given enough time, the subject will certainly guess the target. Thus, the situation seems amenable to a probabilistic analysis that provides continuity between "true" and "chance" detections based on random variables, perhaps along the lines of signal detection theory. Related matters will be considered briefly below.

Does the exponential relationship suggest hypotheses about the search process? It is interesting that a very similar relationship underlies Shepard's hypothesis about the decision process involved in stimulus generalization (e.g., Shepard, 1987). The difference between Shepard's result and the result suggested here reflects the complementarity of discrimination and generalization: For search, the discriminative detection response decreases in probability with similarity, whereas, for generalization, response probability increases with similarity. For the generalization case, Shepard has derived the exponential relationship from general assumptions that an animal might make about whether a novel stimulus is in a "consequential region" containing a reinforced stimulus (Shepard, 1986). His argument is functional and cognitively oriented: "The problem that the individual faces is that of deciding whether the new stimulus, even though different from the preceding one, is nevertheless similar enough that it, too, might belong to the class of objects possessing the same reinforcing consequences"' (Shepard, 1986, p. 60). In this model, response probability reflects the probability that a stimulus is found to be in the appropriate class.

Although both the generalization and the odd-item search tasks involve partitioning stimuli into "same" and "different" categories, it is not obvious how a decision process like that proposed by Shepard might apply to the search situation. In generalization, a test stimulus may clearly differ from a reinforced stimulus and yet be in the consequential region. In search, any difference should contribute to target detection. Instead of being determined by the chance that a stimulus is in a consequential region, RT would seem to be determined by how fast any difference at all can be detected. In short, search would not seem to involve the sort of cognitive decision processes that Shepard suggests for generalization. Furthermore, the most comparable measure for which there appears to be extensive data, namely discriminative reaction time, seems to be related to distance by a reciprocal rather than an exponential function (Shepard, 1981).

More appropriate comparisons may be sought in the search literature, but here, unfortunately, researchers typically have used just two classes of stimuli: confusable and nonconfusable (e.g., D. S. Blough, 1979; Estes, 1972; Farmer \& Taylor, 1980). This dichotomous classification is reflected in models of search as well. A dominant view holds that targets dissimilar from their distractors are identified by fast, automatic, parallel processes, which yield rapid pop-out of the target and an insensitivity of search speed to display size. In contrast, similar target-distractor pairs are said to invoke slower, attention-demanding, serial processing that is sensitive to display size (e.g., Hoffman, 1979; Schneider \& Shiffrin, 1977; Treisman \& Gelade, 1980). It is not clear whether the automatic process is of variable duration or may occur with a short and invariant RT, once some threshold of dissimilarity is reached. For the slower serial process, speed decreases with target-distractor similarity, but little has been done to delineate the details of this relationship.

Research restricted to extreme classes of stimulus similarity could not, of course, yield functional relations of the sort reported here. However, the idea that these classes are processed differently does suggest that such functions, once found, might be of a particular sort. If dissimilar stimuli are processed differently than similar stimuli, the function relating RT to similarity might well differ in the two cases, leading to a discontinuity at the point of switchover from dissimilar to similar. Thus, one might expect to see distinct limbs in the RT-similarity functions of Experiment 1. In fact, there was no indication of such discontinuities, either in the mean data (Figures 5, 8, 11, and 14) or in the data from individual subjects (not shown). The only indication of altered processing when targets and distractors became quite similar appeared in the RT distributions from Experiment 2: Theoretical fits of the data for the most similar stimulus pair were notably poorer than for the other pairs (lower right panel, Figures 15 and 16).

Even if two processes are generally needed to account for search data, there are several reasons why the results reported here might not distinguish these processes. First, the range of similarity may have been insufficient to generate complete RT-similarity functions; we have noted that stimulus pairs leading to high error rates were excluded. Second, variability in the data may have smoothed over the predicted discontinuity in the RT-similarity function. Third, the procedure may not have isolated the hypothesized automatic parallel process. It has been argued that automatic pop-out of a dissimilar target occurs prior to the localization of the target, which requires slower focal attention (e.g., Treisman \& Gelade, 1980). The present procedure requires the pigeon to peck at, and thus to localize, the target. This requirement may necessarily invoke a slow localization process that masks rapid popout regardless of stimulus similarity.

A final reason for the absence of evidence for two processes in the present results could be that pigeons employ only one process. Past work has revealed more similarities than differences between pigeons and humans in the area of search and perception. In search, for example, the RT-display-size function steepens with targetdistractor similarity (D. S. Blough, 1979; P. M. Blough, 1984); the two species show similar patterns of confu- 
sions between alphabetic letters (D. S. Blough, 1985) and mirror images (D. S. Blough \& Franklin, 1985). However, there is some suggestion that feature conjunctions are processed differently by pigeons (D. S. Blough \& Franklin, 1985), consistent with the idea that early processing in these birds is more thorough than in humans. Though highly speculative, this suggestion is also consistent with a single-process interpretation of the present data. We may hope for more data bearing on this matter, for cross-species comparisons, revealing patterns of similarity and difference, have been a key factor in understanding many biological processes.

As we just saw, current models of human visual search are largely inapplicable to the present data because they tend to dichotomize similarity. In contrast, something like a similarity continuum is common to models of discrimination and detection; the "decision axis" of signal detection theory is perhaps the most familiar example (e.g., Green \& Swets, 1966). Starting from the idea that stimulus differences can be represented by the distance between points on such a continuum, discriminative reaction times may be derived in various ways. Often suggested is the random walk, where stimulus differences accumulate over time until a decision boundary is reached (e.g., Link, 1975). Ratcliff (1978) incorporated this idea in an impressive model of memory search, which is of special interest here because same-different memory search presents some of the same problems as odd-item visual search and the two may involve common processes. Interestingly, Ratcliff's model also predicts reaction time distributions of the form found in Experiment 2.

Following these leads, it would doubtless be possible to construct a model of visual search that would yield data like those found here. However, so many processes yield exponential decay that a model tailored to generate only the data presented here could carry little conviction. We hope eventually to state a model that would account as well for other aspects of pigeon search (e.g., display size functions and search asymmetries), and that would also relate effectively to data from human subjects.

\section{REFERENCES}

BLough, D. S. (1979). Effects of the number and form of stimuli on visual search in the pigeon. Journal of Experimental Psychology: Animal Behavior Processes, 5, 211-223.

Blough, D. S. (1984). Form recognition in pigeons. In H. L. Roitblat, T. G. Bever, \& H. S. Terrace (Eds.), Animal cognition (pp. 277289). Hillsdale, NJ: Erlbaum.

BLough, D. S. (1985). Discrimination of letters and random dot patterns by pigeons and humans. Journal of Experimental Psychology: Animal Behavior Processes, 11, 261-280.

BLough, D. S. (1986). Odd-item search by pigeons: Method, instrumentation and uses. Behavior Research Methods, Instruments \& Computers, 15, 413-419.

BLovgH, D. S. (in press). Form similarity and categorization in pigeon visual search. In M. L. Commons, R. J. Herrnstein, \& S. Kosslyn (Eds.), Quantitative analyses of behavior: Pattern recognition and concepts in animals, people, and machines. Hillsdale, NJ: Erlbaum.

Blough, D. S., \& Franklin, J. J. (1985). Pigeon discrimination of letters and other forms in texture displays. Perception \& Psychophysics, 38, 523-532.
Blough, P. M. (1984). Visual search in pigeons: Effects of memory set size and display variables. Perception \& Psychophysics, 35 , 344-352.

BURBECK, S. L., \& LUCE, R. D. (1982). Evidence from auditory simple reaction times for both change and level detectors. Perception \& Psychophysics, 32, 117-133.

EкMAN, G. (1954). Dimensions of color vision. Journal of Psychology, 38, 467-474.

ESTES, W. K. (1972). Interaction of signal and background variables in visual processing. Perception \& Psychophysics, 12, 278-286.

FARMER, E. W., \& TAYLOR, R. M. (1980). Visual search through color displays: Effects of target-background similarity and background uniformity. Perception \& Psychophysics, 27, 267-272.

Green, D. M., \& SWets, J. A. (1966). Signal detection theory and psychophysics. New York: Wiley.

Harris, J. R., Shaw, M. L., \& Altom, M. J. (1985). Serial-position curves for reaction time and accuracy in visual search: Tests of a model of overlapping processing. Perception \& Psychophysics, 38, 178-187.

Harris, J. R., Shaw, M. L., \& Bates, M. (1979). Visual search in multicharacter arrays with and without gaps. Perception \& Psychophysics, 26, 69-84.

HOCKLEY, W. E. (1984). Analysis of response time distributions in the study of cognitive processes. Journal of Experimental Psychology: Learning, Memory, \& Cognition, 10, 598-615.

Hoffman, J. E. (1979). A two-stage model of visual search. Percep tion \& Psychophysics, 25, 319-327.

HoHLE, R. H. (1965). Inferred components of reaction times as functions of foreperiod duration. Journal of Experimental Psychology, 69, 382-386.

Krantz, D. H., \& Tversky, A. (1975). Similarity of rectangles: An analysis of subjective dimensions. Journal of Mathematical Psychology, 12, 4-34.

LiNk, S. W. (1975). The relative judgment theory of two choice response time. Journal of Mathematical Psychology, 12, 114-135.

LUCE, R. D. (1986). Response times: Their role in inferring elementary mental organization. New York: Oxford University Press.

McGil., W. J. (1963). Stochastic latency mechanisms. In R. D. Luce, R. R. Bush, \& E. Galanter (Eds.) Handbook of mathematical psychology (pp. 309-360). New York: Wiley.

Monahan, J. S., \& Lockhead, G. R. (1977). Identification of integral stimuli. Journal of Experimental Psychology: General, 106, 94-110.

Neisser, U. (1967). Cognitive psychology. New York: Meredith.

PoDgorny, P., \& GARNer, W. R. (1979). Reaction time as a measure of inter- and intraobject visual similarity: Letters of the alphabet. Perception \& Psychophysics, 26, 37-52.

Ratcliff, R. (1978). A theory of memory retrieval. Psychological Review, 85, 59-108.

RATCLIFF, R. (1979). Group reaction time distributions and an analysis of distribution statistics. Psychological Bulletin, 86, 446-461.

RATCLIFF, R., \& MuRDock, B. B. (1976). Retrieval processes in recognition memory. Psychological Review, 83, 190-214.

SAS InSTITUTE (1982). SAS system [Computer program]. Cary, NC Author.

SCHNEIDER, W., \& SHIFfrin, R. M. (1977). Controlled and automatic human information processing: I. Detection, search, and attention. Psychological Review, 84, 1-66.

Schönemann, P. H., Dorcey, T., \& KienapPle, K. (1985). Subadditive concatenation in dissimilarity judgments. Perception \& Psychophysics, 38, 1-17.

SHEPARD, R. N. (1957). Stimulus and response generalization: A stochastic model relating generalization to distance in psychological space. Psychometrika, 22, 325-345.

SHEPARD, R. N. (1958). Stimulus and response generalization: Tests of a model relating generalization to distance in psychological space. Journal of Experimental Psychology, 55, 509-523.

SHEPARD, R. N. (1964). Attention and the metric structure of the stimulus space. Journal of Mathematical Psychology, 1, 54-87.

SHEPARD, R. N. (1965). Approximation to uniform gradients of generalization by monotone transformations of scale. In D. J. Mostofsky (Ed.), Stimulus generalization (pp. 94-110). Stanford, CA: Stanford University Press. 
SHEPARD, R. N. (1974). Representation of structure in similarity data: Problems and prospects. Psychometrika, 39, 373-421.

ShePARD, R. N. (1981). Discrimination and classification: A search for psychological laws. Presidential address to the Division of Experimental Psychology of the American Psychological Association, Los Angeles, CA.

SHEPARD, R. N. (1986). Discrimination and generalization in identification and classification: Comment on Nosofsky. Journal of Experimental Psychology: General, 115, 58-61.

ShePard, R. N. (1987). Toward a universal law of generalization for psychological science. Science, 237, 1317-1323.

SYSTAT, INC. (1986). SYSTAT [Computer program]. Evanston, IL: Author.

Treisman, A. M., \& Gelade, G. (1980). A feature-integration theory of attention. Cognitive Psychology, 12, 97-136.

WENDER, K. (1971). A test of the independence of dimensions in multidimensional scaling. Perception \& Psychophysics, 10, 30-32.

Wiener-Ehrlich, W. K. (1978). Dimensional and metric structures in multidimensional stimuli. Perception \& Psychophysics, 24, 399-414.

YANTIS, S., \& JonidEs, J. (1984). Abrupt visual onsets and selective attention: Evidence from visual search. Joumal of Experimental Psychology: Human Perception \& Performance, 10, 601-620.

\section{APPENDIX}

\section{Determination of $K$}

Functions relating reaction time to scaled distance (Figures 5 , 8,11 , and 14) depend on the scaling of the variables on the axes. Distance-the abscissa-is the Euclidean distance in multidimensional space between the points representing stimulus objects, as fitted by ALSCAL. Arbitrary monotonic rescaling was allowed in this process; that is, only the ordinal characteristics of the data were preserved by ALSCAL. Thus, it should make little difference what monotonic transformation of the RT data were input to ALSCAL, and in fact ALSCAL produced very similar results for all experiments, regardless of whether linear or log-transformed data were used.

The monotonic transform found by ALSCAL to yield the best fit to distance was approximately exponential. The transformation of Equation 1 renders this a linear function (Figures 5, 8, 11 , and 14 ), but assumes a constant value $K$. As the analysis of Experiment 2 indicates, $K$ may be taken to represent a fixed component of RT or, more exactly, the mean of a fixed distribution with which an exponential distribution of search times is convolved. Experiment $2 \mathrm{~B}$ was an attempt to estimate this fixed distribution. However, the mean of this distribution could not be used for $K$ in the $\log (\mathrm{RT}-K)$ transform for all of the experiments because minimum RT appeared to drift slightly over the many sessions during which the experiments were run. Thus, for example, the minimum RT obtained for "easy" searches in a few cases in Experiment 1 was a little below the $K$ determined in Experiment 2B.

Experiment $2 \mathrm{~B}$ was run in just 5 sessions immediately after Experiment 2A. Thus, we may assume that the $K$ for each subject from Experiment 2B applies reasonably well to the data for that subject from Experiment 2A. The difference between the fastest true search (i.e., with many forms on the screen) in Experiment $2 \mathrm{~A}$ and the minimum determined in Experiment $2 \mathrm{~B}$ averaged $50 \mathrm{msec}$. On this basis, the value of $K$ for each of the other experiments was estimated for each subject by subtracting $\mathbf{5 0}$ msec from the lowest mean RT obtained from that subject:

$$
K=\min \mathrm{RT}-50,
$$

where min RT is the minimum RT in msec actually obtained in a given experiment. This estimate is doubtless somewhat ques- tionable, but variations in this value between 10 and $100 \mathrm{msec}$ had rather little effect on the functions displayed here.

\section{Estimation of RT for Search Failures}

For the great majority of stimulus combinations used in these experiments, the target was responded to within the $8.5 \mathrm{sec}$ allowed for each trial. Across Experiments 1A-1D, $92 \%$ of targetdistractor combinations yielded such "hits" on $75 \%$ or more of their presentations. For the most similar stimulus pairs, however, search often failed within the allowed $8.5 \mathrm{sec}$. The RTs for these arrays form a truncated distribution whose mean presumably underestimates the mean of a distribution that would have been obtained from the use of very long trials.

It is possible to correct the mean RT for difficult stimulus arrays by estimating the missing tail of the RT distribution. One assumes, with the support of Experiment 2, that the tail of the RT distribution is approximately exponentially distributed. The argument then rests on two facts about the exponential distribution: (1) The area under the distribution beyond a given cutoff time is simply related to the decay constant, $\lambda$, of the distribution (e.g., McGill, 1963, p. 318), so percent "misses"' can be used to estimate $\lambda$. (2) The mean of the distribution is $1 / \lambda$, and the mean of the area beyond the cutoff time is $t_{c}+1 / \lambda$. Here, the cutoff time is measured from $K$, the "irreducible minimum" $\mathrm{RT}$ at which the exponential component is assumed to commence. The derivation of $K$ is given above. Thus, for each missing RT for a given array we can substitute the mean RT of the missing tail for that array:

$$
\begin{aligned}
F(\mathrm{t})= & \lambda \mathrm{e}^{-\lambda \mathrm{t}} \\
A= & \mathrm{e}^{-\lambda \mathrm{t}_{\mathrm{c}}} ; \lambda=(-\log \\
& A) / \mathrm{t}_{\mathrm{c}} \\
M= & \mathrm{t}_{\mathrm{c}}+1 / \lambda,
\end{aligned}
$$

where $F(\mathrm{t})$ is the exponential distribution of RTs, $A$ is the relative area of the missing tail, or percent "misses," $\lambda$ is the exponential decay constant, $\mathrm{t}_{\mathrm{c}}=8.5-K$, and $M$ is the mean RT in the tail.

Although this correction for misses seems reasonable for low miss rates, it is questionable for high rates. If the probability of detection is zero, the exponential formula predicts an infinitely large RT. However, zero probability should not be assigned, even to an impossible search among identical objects, because of the occurrence of correct guesses. We estimate that such correct guesses occur with our procedure, within $8.5 \mathrm{sec}$, on approximately $15 \%$ of impossible search trials. (This estimate is based on unpublished work that presented birds with such impossible searches.)

As the number of misses becomes large, RT becomes progressively more dependent on estimated rather than real values, and guesses presumably play an increasing role. Rather than dealing with the resulting questionable estimates, the RT - distance functions displayed here omit points that represent stimulus pairs successfully searched on fewer than $75 \%$ of trials. However, since multidimensional scaling required some estimate of these extreme RTs and used only ordinal relationships, which might not be too distorted, they were included in the multidimensional scaling process.

\section{Effect of Noise on Scaling Solutions}

It is argued under Experiment 2 that obtained RT consists of two components, one governed by stimulus factors such as similarity, the other by motor response time and other nonstimu- 
lus factors. This argument implies that the stimulus-governed RT component approaches zero with increasing stimulus dissimilarity. However, as it shrinks, this RT component may approach the variance of the second component. When this happens, random influences begin to disturb the order of mean RTs obtained from different pairs of very dissimilar stimuli. To the extent that this happens, monotonic rescaling cannot yield the appropriate underlying structure.

Very low RTs, corresponding to large distances in psychological space, may thus tend to be disordered by variability. In attempting to fit such disordered points, ALSCAL tends to equalize long distances. To do this while still providing good fits at shorter distances, the algorithm curls the representation of the points into new dimensions. For an essentially one-dimensional measure disordered in this way, the best-fitting representation becomes the $\mathrm{C}$-shaped configuration in two dimensions (Shepard, 1974), since this permits correct local distances but tends to equalize longer ones. Several of the data sets from Experiment 1 showed this sort of curl and yielded intuitively satisfying spatial representations in more dimensions than might have been expected.

Can such noise alter the functional relation between RT and distance? A partial answer to this question is provided when artificial data sets with known data-distance functions are perturbed before their structure is recovered through multidimensional scaling. A set of simulations tested the hypothesis that the apparent exponential RT-distance function might be generated from an underlying linear relationship that was subject to a lower bound. Noise from a normal deviate was added to truncated data (simulating a limit or asymptote) that were generated from onedimensional distances. One-dimensional scaling produced somewhat distorted distances; two-dimensional fits worked well, producing the $\mathrm{C}$-shaped configuration described above and better representing local distances. In both cases, however, ALSCAL recaptured the linear distance-data function rather clearly, with only unsystematic perturbation.

\section{The Influence of Metric Structure}

A Euclidean psychological space has been assumed throughout this article. ALSCAL used Euclidean distances among stimulus objects to arrive at a best-fitting configuration, and Euclidean distances among objects in the final configurations defined interobject similarity. However, it may be that the assumed Euclidean space is not appropriate, especially for some sorts of stimuli. It has often been maintained, for example, that the cityblock metric is more appropriate for stimuli with separable dimensions (e.g., Shepard, 1964). Perhaps the inappropriate assumption of Euclidean space generates misleading data-distance functions.

To test this possibility, alternative metrics were explored. First, the data sets from Experiments 1B, 1C, and 1D were scaled using another scaling program called MDS (SYSTAT, 1986) that permitted the use of Minkowski exponents $(r)$ other than the Euclidean $(r=2)$. The data were scaled in two, three, and four dimensions using $r=1$ (city-block), $r=1.5$, and $r=2$. The results across these $r$ values varied rather little in spatial configuration, stress, and the shape of the RT-similarity function. In every case but one, stress for the Euclidean $(r=2)$ fit was lower than for the city-block $(r=1)$ fit. The exception was for the data of Experiment 1C (separable dimensions) where stress in two dimensions was slightly lower for $r=1(.076)$ than at $r=2(.079)$; this difference was reversed in three dimensions ( .056 for $r=1, .048$ for $r=2$ ) and four dimensions ( .051 for $r=1, .024$ for $r=2$ ). For these data, $r=1$ also yielded a slightly less linear function than $r=2$ in two and three dimensions.

In addition, a number of simulations were run with either 16 or 26 objects distributed in regular or random configurations in two- or three-dimensional spaces, with distances defined either by the Euclidean formula $(r=2)$ or by the city-block formula $(r=1)$. These distances were then input to ALSCAL, either as computed or after exponentiation. Despite the use by ALSCAL of the Euclidean distance function, the spatial configurations were in general recreated quite closely for the various inputs. The relationship between the data and the original distances was also rather well reflected in the relationship between the data and the computed distances, though city-block data induced noise around this function, and also induced some curvature opposite in sense to that reflected in the basic exponential relationship.

In summary, rescaling suggested that the Euclidean assumption was generally appropriate for these data sets, and both rescaling and simulation suggested that the RT-similarity relationship was not severely dependent on the assumed metric.

(Manuscript received March 26, 1987; revision accepted for publication July 13, 1987.) 